the corpus luteum is present in the ovary. Where ovulation has not taken place, menstruation, though occurring at the normal time, takes place from an undeveloped resting endometrium. It is thus necessary to suppose that whereas the pre-menstrual development of the uterus is dependent, as would be expected, upon the presence of the corpus luteum, the actual factor causing necrosis and breakdown of the epithelium is not set in action by the atrophy of the corpus luteum, although operating at the same time, and is independent of any morphological cvarian cycle.

Evidence has lately become available as to the site of origin of the substance concerned. Some years ago it was shown by Allen that menstruation could occur in ovariectomized monkeys after a series of injections of oestrin. Since the hormone is associated with the follicular phase of the cycle in the normal animal, these experiments appeared to simulate the condition of affairs found in the non-ovulating monkey. Very recently, however, Hartman has shown that this effect of injection of the ovariectomized monkey is completely lacking if the animal is hypophysectomized. It is thus necessary to suppose that Allen's results were due to activation of the pituitary body by the injection of oestrin, and that the substance causing necrosis of the endometrium is probably hypophyseal in origin.

The importance of studying the nature of this factor causing necrosis has been emphasized for some years by Shaw, and it seems probable that further progress with the study of the mechanism of the human menstrual cycle will be dependent upon some working hypothesis of the factor involved in the necrosis of the endometrium. From the point of view of the subject under discussion, namely, the factors concerned in menorrhagia unaccompanied by uterine disease, it is quite evident that this necrosis factor is of prime importance. In cases of menorrhagia, the morphological cycle in the ovary may be abnormal or even entirely suppressed, and a condition may be found analogous with that in the non-ovulating monkey, except that the period of destruction of the endometrium is prolonged indefinitely. This would appear to point to the possibility that the factor causing necrosis loses its normal cyclic activity and functions persistently. If such an explanation can be substantiated, an important basis will have been found for the experimental attack upon the problems of menorrhagia.

\section{VARICOSE ULCER*}

BY

A. DICKSON WRIGHT, M.S., F.R.C.S.

ASSISTANT DIRECTOR, SURGICAL CNIT, ST. MARY'S HOSPITAL; ASSISTANT SURGEON, PRINCE OF WALES HOSPITAL (With Special Plate)

The femoral artery delivers a very large quantity of blood to the leg every twenty-four hours, probably more than a thousand pints. The arterial blood is delivered with all the force of the heart and gravity behind it, but when
Of this group, it is fairly certain that the last two factors are the most important. Should the veins becomo incumpetent to return the blood to the heart from blockage, atony of their muscular coats, or failure of the valves, serious stagnation phenomena will result, and that naturally in the region most remote from the heart, because here the effect of gravity is at its maximum. The support of shoes and boots and the pressure on the soles prevent oedema and stagnation in the feet. Figures 1,2 , and 3 show the regions in which ulcers develop according to the type of footwear worn. The upper confine of this ulcer-

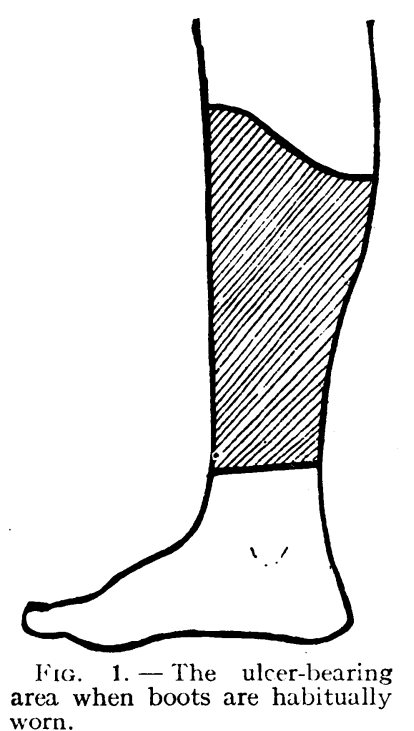

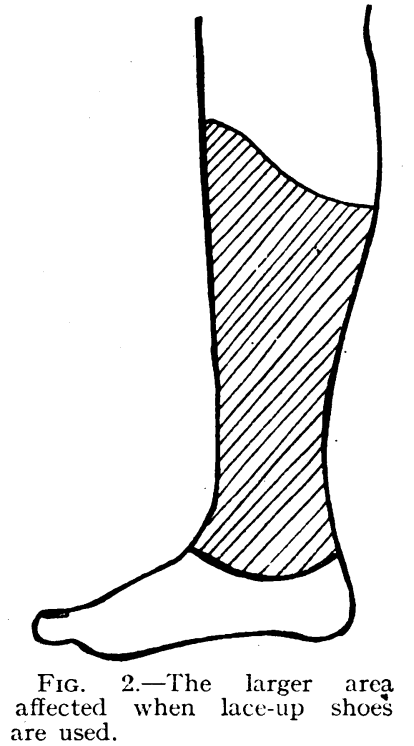

affected when lace-up shoes are used. this force is dissipated in the capillary bed there comes into play a wonderfully complicated mechanism whereby this large volume of blood is returned to the heart against gravity. Let there be a drop in efficiency of one part in two hundred in this return mechanism, and serious changes will occur in the leg.

Many factors assist in the return of the venous blood, such as the elasticity of the skin, the contraction of the leg muscles, abdominal and thoracic aspiration, vis a tergo of capillary pressure, muscular tone and contractions of the vein walls, and the elaborate valving of the veins.

* Read in opening a discussion in the Section of Dermatology at the Annual Meeting of the British Medical Association, Eastbo irne, 1931 . ative region is constant, and the lower corresponds to the limits of foot support. By a simple consideration of this area which carries probably 99 per cent. of the indolent ulcers affecting mankind, we see both the cause and the proper line of treatment. The final cause in all cases is gravity, which is too much for the legs of erect-standing mankind, and the best treatment is constant support.

The name varicose ulcer is an oxymoron, and is not comprehensive enough, because many of these ulcers have other causes, such as venous thrombosis, ankylosis of joints, paralysis of the leg muscles, lymphatic blockage, etc. More comprehensive descriptions are: ulcus cruris, indolent ulcer of the leg, chronic leg ulcer, stasis ulcer, 
static ulcer, and the term that I have suggested, namely, gravitational ulcer.

The necessity for support for swollen legs was probably discovered many hundreds of years ago, and it is impossible to trace the originator of this very obvious principle, which, nevertheless, seems to be forgotten by so many. A study of the vast literature on this subject reveals literally hundreds of methods of treatment advocated, which neglect this one vital essential to every line of treatment. All efforts seem to have been concentrated on finding a magical salve, powder, or even gas, which would stimulate waterlogged tissucs iuto healing. Nearly every chronic disease, and many acute ones, have some mechanical obstacle, which hinders Nature in her powers of overcoming infection, and the removal of this obstacle should be the first consideration in every such disease whether it is the muscle spasm of gonorrhoeal ophthalmia or anal fissure, or the faulty hydraulics of a " milk leg."

Treatment of Chronic Ulcer of the Leg

Chronic ulcer of the leg has certain features which impose limitations to the type of the treatment employed. First, its most common complications are poverty and fecundity, so the treatment must be such as not to involve hospitalization, as the mother of a poor-class family must look after her children; and, moreover, it must not be too expensive. Secondly, the disease is very common, and it requires many doctors to treat all the cases in existence, so that the treatment must be simple and within the reach of all medical practitioners, and not too laborious and time-consuming. It is of the utmost importance that the treatment of varicose ulcer should not be left to district and out-patient nurses. Thirdly, the treatment must not be too tiresome or painful, or the patient will prefer the disease to the treatment; and if the treatment is bravely borne, only for the ulcer to relapse, the recurrence assumes the dimensions of a calamity in the mind of the patient, who then becomes sceptical of all kinds of treatment.

The treatment I adopt for this difficult disease is simplified as far as possible. It is ambulant, and the patient's employment is not interrupted. The visits to hospital are at intervals, on the average, of three weeks. The ulcer is dressed with nothing but its own discharge, so that unnecessary labour and expense are avoided; more important, the efficacy of the support is not interfered with by the repeated necessity of dressing the ulcer. The bandage used to support the leg is of elastic sticking plaster, so that it cannot move when once applied, and it adjusts itself to the shrinkage of the leg. Another advantage of this adhesive plaster is that it is, to a certain extent, waterproof, allowing restricted bathing, and, more important, keeping the discharge in, and thus saving changes of dressing. Owing to the elastic nature of the bandage, it can be wound on smoothly in a continuous spiral; this takes much less time than the method of applying separate strips of ordinary adhesive. In large ulcers, skin grafts are buried in the granulations by insinuation of small implants, by darning in threads of skin, or by injecting suspension of skin in saline, but this makes no difference to the method of dressing. The sticking plaster is used just the same, and the skin grafts seem to be invariably successful.

All through it is remembered that the ulcer is not the condition being treated, but an underlying stagnation of the venous blood, lymph, and tissue fluid. If this is due to varicosity of the veins, these are treated by injections of sodium morrhuate.

Ligations of large penetrating veins and venous crist: are done in severe cases, because the cure of these cases by injection is very difficult, and recurrence of the varicosity common. These ligations are performed in the out-patient department, as the whole treatment must be ambulatory, no beds being available in hospital for these cases. In the other cases, where there is venous obliteration, or other cause of indolent ulcer, steps must be taken to ensure that the patient will have continuous support over a long period of time.

Here arises the very important question of the treatment of the varicose veins which develop after deep thrombosis of the puerperium, typhoid fever, etc. It is customary to abstain from injections in these cases, but $I$ have gradually ignored this contraindication, and have now treated 56 cases of ulcer associated with old "white leg" ; in 42 of these, large varicosities were injected, and in the remaining $\mathrm{I}_{4}$ there were not present any varicose veins. In addition to these, a large number of similar cases have been injected suffering from periphlebitis or swelling, but I have never seen any damage result in the shape of increased swelling, cyanosis of the limb, etc. In cases of damage to the deep circulation, I regard these superficial varicose veins as an added embarrassment to the sound veins which are transmitting blood in the upward direction.

\section{Contraindications}

As regards contraindications to this method of treatment, there are only two-namely, associated arterial disease and diabetes. I have observed (and this is a most important point) in legs which have been damaged by long-standing venous stagnation that, if gangrene supervenes as a result of senile or diabetic arteritis, it is not the toes which are affected first, but the area of skin outlined in Figures $I, 2$, and 3. Treatment by compression would in these cases be disastrous, and one must always be on the look out for them. The other complications of varicose veins-eczema, phlebitis, periphlebitis, pedal deformity, periostitis, arthritis, etc.-are nearly all benefited by this treatment, and their presence should not dissuade one from practising any part of the treatment-compression, injection, or skin grafting.

\section{Disadvantages}

The disadvantages of this method of treatment are:

Failure.-Many cases are referred to me because the treatment has been ineffectual, but $I$ have never failed to bring about rapid healing in these cases. The almost invariable cause of failure is the looseness of the bandage; the patient is always astonished at the force I employ, and delighted with the relief afforded. Another cause of failure is the delegating of the bandaging to those who do not understand the principle of the treatment. In one case referred to me of a very severe ulcer, following a badly placed incision for cellulitis of the leg, in which $\mathrm{I}$ was informed that my type of treatment had done more harm than good, I learned that the adhesive plaster had been applied on the leg above the ulcer by the out-patient sister, the ulcer and foot being left out to swell, in order that hot fomentations could be applied four times daily to the ulcer. Other cases of failure $I$ have seen have been clue to syphilis or malignant disease, and I have come to regard the lack of response of an ulcer in the former case to be more certain than the Wassermann reaction in making a diagnosis. In malignant disease, of which $I$ have seen two cases, failure to react to the treatment made the aiingnosis clear.

Eczema develops under the adhesive in about 5 per cent. of cases, and seems to be an idiosyncrasy. In about $I$ per cent. of cascs the eczema becomes generalized, affecting chiefly the face and arms. The idiosyncrasy may be to rubber, zinc, or to the morrhuate injections. It is a serious disadvantage when it occurs, especially when it is of the exudative type, and causes great dismay to the patient. I have only once stopped treatment on this 
account, and in that case the patient was in a lunatic asylum. In many cases I have persevered in the face of distressing eczema, with the most gratifying results. Per contra, I have found the most severe "stocking" eczemas, which had been treated by other methods for a long time, immediately improve with elastic compression. I have had adhesive bandages made, impregnated with rivanol $\mathrm{r} / \mathrm{I}, 000$, ichthyol 5 per cent., aluminium acetate 5 per cent., for these cases, and a change to one or other often checks the development of an eczema.

Blisters develop under the bandage, and burst and ooze quantities of fluid. They are often on the sole of the foot, and cause a fair amount of pain and discharge, and the base looks as if it would form a new ulcer, but I have not found that this occurs. I never stop the treatment because of the development of blisters.

Cutting of the bandage into the skin produces linear ulceration, and is due to faulty bandaging in very oedematous legs. It is avoidable by placing longitudinal strips up the leg as splints, and by overlapping each turn of the bandage two-thirds of its width by the ensuing turn.

Pain is sometimes increased at first by the pressure. I find this in only io per cent. of cases, the remaining 90 per cent. of the patients being very much gratified by the comfort afforded. The pain may be due to blisters or faulty application of the bandage, or to the injections. In certain cases it is in the bones or joints adjoining the ulcer, and in some cases in the ulcer itself ; to avoid this latter pain I increase the pressure over the ulcer by sorbo rubber pads, with adhesive on one side, stuck on over the ulcer on the bandage and then pressed into the ulcer bed with another turn of the bandage. These pads are especially valuable in treating ulcers lying in the sulcus under the malleoli where the bandage does not grip very tightly. The sprinkling of aspirin powder on the floor of the ulcer I have found an excellent analgesic, lasting from four to seven days. Finally, a night powder of a barbiturate and aspirin is a great and justifiable help at the start of the treatment. It saves the patient from worrying during the long night hours over an ulcer, the constant companion for many years, now cut off by stickingplaster. She has fed the ulcer with salves, antiseptics, lint and gauze for so long that she thinks that terriblc things are happening under the bandage.

\section{The Mental Attitude of the Patient}

The mental attitude of the patient is sometimes diffcult, and in nearly all cases has a financial background. In these days of humane government, chronic bronchitis in the husband and a varicose ulcer in the wife will maintain a family in certain parishes, and these valuable diseases will not be parted with too easily. Again, pensions are awarded for ulcers acquired in the Army, and there is a certain type of man who will on no account give up this premium on ill-health. In Central Europe large ulcers of the leg are exposed in the streets for begging purposes, but this is only a degree more degrading than other ways of using ulcers as a source of income. Fortunately, I have found this type of thing very rarely, and then nearly always in men; as a rule, every patient is desperately anxious to get the ulcer healed.

\section{Expense and Difficulty of Treatment}

Expense of treatment should be a very minor consideration in this disease, which is of great economic importance. I have scores of cases of men-mechanics, painters, and so on-who have lost in wages hundreds of pounds, and have cost the Government, health clubs, and hospitals equally large sums of money; these men were all very anxious to get to work and maintain their families. The bandages are fairly expensive, and the 75 pints of sodium morrhuate which I inject annually cost a certain amount, but it is found that the patients' contributions more than cover the costs. A great argument against too cheap a treatment is that it will fall into the hands of nurses, and we shall have a repetition of Unna's bandages applied year after year to the same quota of patients, without any effect at all.

Difficulty of the treatment is used as an argument, but if good bandaging and injection technique are once learned, it is very easy, and the knowledge so acquired is useful in many other ways. I have found that those who have mastered the method by attending the clinic have always succeeded in getting the same excellent results themselves.

Relapse is a most irritating argument against any treatment. It is better to be without a disease for even a short time than to have it all the time; moreover, in this disease it is easy to prevent the recurrence, if it is anticipated. In 525 cases of cured ulcer, I have only had 18 relapses (3.4 per cent.) in the last three years. None of these was difficult to treat.

The following cases illustrate the method of treatment of two typical difficult cases:

CASE I

For cight years Mrs. R. suffered from ulcer below the internal malleolus of the left foot. It was small and deep, the size of a shilling, but intensely painful, the type of ulcer known as Corlette's irritable ulcer. Owing to the pain the patient walked on the outer border of the front part of the foot, and had a fixed talipes equino-cavo-varus deformity as a result. She was sent to me because the practitioner could make no headway with elasto-plastic bandaging, or any other treatment. There had been a tendency not to persist with any single line of treatment long enough for it to do good, and I felt that it was the patient, a strong-minded woman, who was ordering the treatment. No varicose veins could be seen or felt, and the leg did not seem greatly swollen. A bandage was wound on with a pressure that exasperated the patient, and a small rubber adhesive pad laid over the ulcer and bound tightly on to it, a sleeping powder was prescribed, and the patient returned in a week. She had been much more comfortable, and the ulcer was now flush with the surrounding skin, and definitely healing. The pressure had pushed a lot of oedema out of the leg, and a varicose saphenous vein was now injected casily. The bandage was re-applied as tightly as before, and the sleeping powder omitted. The next time a small deep vein was injected above the ulcer, and in four weeks the ulcer was healed firmly, the total cost of materials being twelve shillings. The support is being kept up by a bandage for six weeks; an elastic stocking will follow, to consolidate the cure, and finally the question of operation for the deformity will have to be discussed with the patient.

\section{CASE II}

Mrs. C., aged 51, and mother of nine children, had for five years suffered from swelling and ulceration of the right leg. She could not recall having either " white leg " or varicose veins. The ulcer, starting from an abrasion, had gradually grown to an immense size.

The area was 67 square
inches, and the girth of the leg 15 inches at the upper

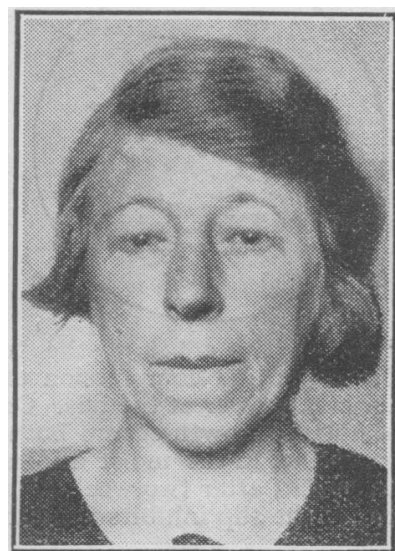

Fig 4-The ulcer facies. margin of the ulcer. (Plate, Fig. 1.)

Fier suffering had been terrible, and she appeared worn out with constant pain and sleeplessness. The exhaustion showed itself in her face, giving her what I have called the "ulcer facies" (Fig. 4). The discharge from the ulcer was most profuse, and this constant albuminous drain.had led to a profound secondary anaemia, with a haemoglobin percentage 
of 35. The Wassermann reaction was negative. The ulcer showed an almost pure culture of $B$. pyocyaneus, and the discharge from the ulcer had no inhibitory effect upon the growth of the organisms. A test with milk showed the presence of trypsin in the discharge. The margins of the ulcer were stony hard, and there was only a narrow strip of skin connecting the skin of the foot with that of the upper part of the leg.

Without any delay, although the ulcer was so foul, it was bound tightly with adhesive plaster. Strips were laid first as shown in Figure 5, to stop the spiral bandage cutting furrows

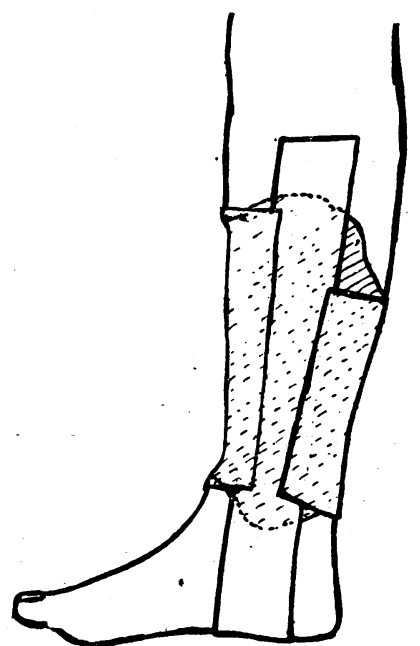

Fig. 5.-Longitudinal strips put on before the spiral comrressing bandage: I. To prevent the cutting of. grooves in the oedematous leg. 2. To prevent the bandage from slipping out of position on the surface of the ulcer which is too moist for the bandage to adhere. This precaution is not necessary when the ulcer is small or the oedema moderate.

in the oedema. A great pressure was used, and, as usual, the patient complained that "she could never stand it." At weekly intervals the bandage was removed (by the patient) and renewed; on four occasions skin was removed from the forearm and slipped under the granulations, once by darning, and three times by implantation.

Although this ulcer was so large, it continued to heal steadily and smoothly, and at the end of thirteen weeks was com-
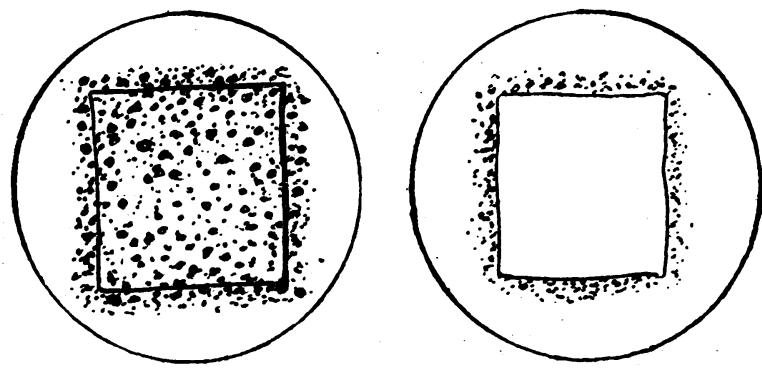

Fig. 6.-The effect of the treatment by retention of the discharge and compression of the whole leg upon the bactericidal power of the pus. On the left, before treatment an abundant growth occurs both in the dried discharge round as well as in the moist discharge under the cover slip. On the right, after two weeks of treatment the moist discharge under the slip inhibits the bacterial growth.

pletely epithelialized after the application of ten bandages, and the girth of the leg was reduced to twelve inches where it had leen fifteen inches (Plate, Fig. 2). With the healing of the ulcer, the discharge diminished, and from the start of the treatment the pain ceased, sleep came, and the lines of pain disappeared from the face; the weight increased by 28 pounds, and the haemoglobin rose to 85 per cent. (Dare).

Sir Almroth Wright investigated the discharge under the adhesive dressing, and found that it now inhibited the growth of the organisms and contained no trypsin. Fig. 6 shows diagrammatically the effect of culture on the discharge. The culture was made by placing a drop of the discharge on a cover slip, and placing it on the surface of medium in a Petri dish. The discharge which oozes out at the edges inspissates, and so its contained leucocytes die, whereas the discharge under the slide remains moist and its leucocytes active. At the edges one has necro-pyo-culture, and under the slide bio-pyoculture.

The remarkable point about this case is that at no time were any varicose veins discovered, and there was no definite history of femoral thrombosis. One can only infer that some change occurred in the venous return of the leg, resulting from the repeated pregnancies, and a vicious circle was inaugurated, resulting in the advanced ulceration.

The value of the term " gravitational ulcer" is illustrated by a case of this kind. A point of interest is that this patient had refused many requests for amputation.

\section{Conclusion}

Loss of circulatory equilibrium in the leg inaugurates a train of pathological changes in a certain well-defined area, where the effect of gravity is at its maximum in the unsupported lower extremity. These changes are numerous, and can be summarized as follows:

Oedema.-(a) Occurring towards the end of the day and pitting on pressure; $(b)$ permanent oedema which still pits on pressure; $(c)$ elastic oedema when the skin is tightly stretched, and there is no pitting on pressure; (d) indurated oedema which is the final stage, and is frequently associated with ulcer.

Changes in the Blood.-(a) Increase of lactic acid and carbon dioxide; (b) decrease in oxygen content.

Changes in the Skin Structures.-(a) Loss of hair on the leg; (b) drying up of secretions of sweat and sebaceous glands.

Inflammatory Changes in the Skin.-(a) Eczema of dry, scaly, or exudative type; (b) septic blisters of skin; $(c)$ pruritus; $(d)$ dermatitis artefacta from the scratching : (e) erysipelas.

Ulceration involving (a) skin; (b) subcutaneous tissue; $(c)$ tendons and muscle very rarely; $(d)$ bone, even more rarely.

Changes in Subcutaneous Tissues.-(a) Inflammation in the neighbourhood of the veins-periphlebitis-which is very common, and often miscalled phlebitis; $(b)$ fibrosis, which is always present in ulcer cases; $(c)$ suppuration: (d) ossification, which may take place in the bed of the ulcer, but more usually throughout the whole of the devitalized area in patches; $(e)$ calcification.

Gangrene in the ulcer-bearing area when arterial super venes upon venous disease.

Changes in the Muscles.-(a) Brown degeneration and atrophy; $(b)$ ossification and calcification; $(c)$ contractures from fibrosis.

Changes in the Bones.-(a) Periostitis, which more commonly affects the fibula for some unknown reason; $(b)$ osteitis, with rarefaction and cystic areas in the bone.

Changes in Joints.-(a) Atrophic arthritis; (b) osteoarthritis ; $(c)$ ankylosis.

Changes in the Veins.-(a) Rupture; (b) phlebitis, which is occasionally suppurative.

Changes in Fascia.-Usually ossification of interosseous membrane and other intermuscular septa.

Changes Secondary to Clceration.-(a) Malignant (Marjolin's) disease, which seems to be much more common in men; (b) tetanus; (c) deformities of the foot and knee, resulting from faulty posture in walking in an endeavour to relieve pain, producing pes cavus, equinus, valgus or varus, alone or combined. This deformity is at first spastic, and disappears when the pain goes, but later on the muscles become permanently contracted. I have 
twice done tendon-lengthening operations for this type of deformity. Deformity resulting from fibrosis of the ulcer bed generally produces talipes equinus and calcaneụs; (d) mental changes, chiefly melancholia, neurasthenia, and hebetude.

\section{COMMENTARY}

This is a very formidable list of complications to which rarities can still be added. They all result from loss of balance between arterial and venous systems of the leg. This balance can be restored in two ways-by lying down and by reinforcing the skin with an elastic support. In the cases due to varicose veins a great improvement can be made by obliterating, and in bad cases also ligating, the varices, so that 75 per cent. of ulcer cases can be cured, and remain cured, without any support but the elasticity of the patient's own skin.

Ulcers of the leg will always be with us till we find a way of preventing femoral thrombosis after operations, fractures, and childbirth ; but they will diminish in number because of the simplicity of the injection treatment for early varicose veins. By this method of treatment we have a certain way of curing all gravitational ulcers. The treatment of small ulcers, up to 4 square inches, is a very simple matter, quickly accomplished, with a minimum of trouble. These ulcers can be cured by other methods, I admit, but generally at the expense of much trouble and time to patient and surgeon. The larger ulcers are generally acknowledged to be incurable, but they can be readily cleared up by this method, even when of the gigantic dimensions of $\mathrm{I} 8 \mathrm{o}$ square inches ( $\mathrm{I} \frac{1}{4}$ square feet!), the largest ulcer I have yet treated since I have kept statistics. I have now 525 cases of cured ulcer of a total area of a little over 25 square feet.

One of the most wonderful things in nature is surely to see the ulcer which started a quarter to half a century ago (my longest case is fifty-four years) begin to throw up its granulations, flatten out its margins, pull in its edges, and epithelialize in the way described so well by the "Father of Surgery"-John Hunter-many years ago.

\section{A CASE OF ACHALASIA OF THE CARDIA}

$$
\text { BY }
$$

H. B. BUTLER, F.R.C.S.ED.

SURGEON, ROYAL SURREY COUNTY HOSPITAL, GLILDFORD (With Special Plate)

The following is the history of a male, B. K., aged 35 , who was sent to the $x$-ray department of the Royal Surrey County Hospital with a probable diagnosis of pyloric obstruction.

There was a history of difficulty in "swallowing " for the past two years, much worse during the last four months. He had lost about a stone during the latter period, but though rather emaciated his physical condition was fairly good. $\mathrm{He}$ was of the opinion that " all " his food returned after having been retained for some hours; it gradually worked up, but he did not actually vomit. He got a feeling of tightness and great discomfort in the chest until the food was regurgitated. He had been given a barium meal at home, six hours before being seen at hospital, and when he was examined on the screen it was seen that this meal was still in the greatly distended oesophagus, none being seen in the stomach or elsewhere. The obstruction was at the cardia.

The oesophagus was evacuated, and then a soft mercury tube was passed into the stomach without any obstruction being encountered. Immediately after the passage of the tube he was given half a pint of beaten egg and milk, which he swallowed with ease and evident pleasure, saying that it was the first food he had felt entering his stomach for months. He was admitted to hospital to be taught to pass the tube himself, which he soon accomplished, passing it before every feed; and he was discharged in about a week, able to take all soft food and doing well.

On June 14th, 1929, he came again, and complained of difficulty in swallowing, but as the tube was seen to pass easily into his stomach he was told to persevere (see Plate, Fig. 1). On July 27th he was readmitted, his condition then being nearly as bad as when first seen. Fig. 2 shows an immediate meal distending the oesophagus (July 27th). Fig. 3, taken on July 31st, shows a mercury tube, which he was able himself to pass into his stomach. Fig. 4 shows a bread-and-milk barium meal, the consistency of cream, taken immediately after the successful passage of the tube, and demonstrates the fact that the achalasia was immediately present, even directly after dilatation with a large-sized tube.

No obvious cause for the condition could be discovered, and the patient begged for " an operation, and be done with it." I had had no experience of dilatation with an inflatable bag, and felt that operation offered the best chance of success.

Operation was performed on August 1st. No cancer or ulcer of the stomach or duodenum was found, nor any lesion of the gall-bladder. A transverse incision in the anterior wall allowed three fingers to be introduced into the stomach, but to my surprise I had great difficulty in locating the cardia. (In this connexion I was afterwards interested to read in a paper published by the Mayo Clinic: "The silk thread is just as valuable a guide to manual dilatation from below as it is to dilatation from above, by means of the hydrostatic dilator."') When the cardia was found, one finger was introduced, then a second, and then a third, before there was any sensation that further dilatation would be dangerous. There was no palpable hypertrophy. The stomach and the abdominal wound were then closed. The patient made an uninterrupted recovery, and left the hospital within three weeks.

As I had no report about this patient I wrote to him, and received a reply dated April 17th, 1931: "I am pleased to inform you that I am still going strong and can eat anything that comes along, from beef-steaks, eggs, and bacon to meat pies, in fact $I$ have not found anything yet that I cannot swallow. Twelve months ago I secured light employment and have continued to work regular ever since, and have had no need to visit my doctor."

There seems to be little doubt that, in the large majority of cases, a cure can be obtained by forced dilatation. In approximately 700 cases at the Mayo Clinic dilatation has been carried out readily and satisfactorily from above, by means of the bag, only three requiring dilatation from below. ${ }^{1}$ On the other hand, Hurst ${ }^{2}$ finds that the far simpler treatment, by means of the mercury tube, is effective in the majority of cases, and only reports one case in which no lasting improvement occurred. In this case forcible dilatation by the bag proved equally unsuccessful, and the patient was operated on by Rowlands, who divided the cardiac sphincter in exactly the same way as the pyloric sphincter is divided in Rammstedt's operation for the hypertrophic pyloric stenosis of infants, the operation curing the condition, which had been present for seven years. If treatment is undertaken by means of a bag, it would obviously be an advantage if the bag were opaque to $x$ rays, so that dilatation could be carried out under direct observation and its correct position verified.

A. M. J. Wright, in the Medical Annual, 1930, extracting from H. P. Mosher's Semon Lecture, says: "The author employs dilatation with an inflatable bag under manometer control, the bag having barium implanted in four strips in its wall, so that the dilatation can be carried out under direct observation with the $x$-ray screen.",3

References

1 Mayo Clinic Collected Papers, 1928, p. 3.

2 Hurst: Medical Essays and Addresses.

'Journ. Lary'ngol. and Otol., March, 1930, 161. 
JAMES YOUNG: MENORRHAGIAS NOT DUE TO UTERINE DISEASE

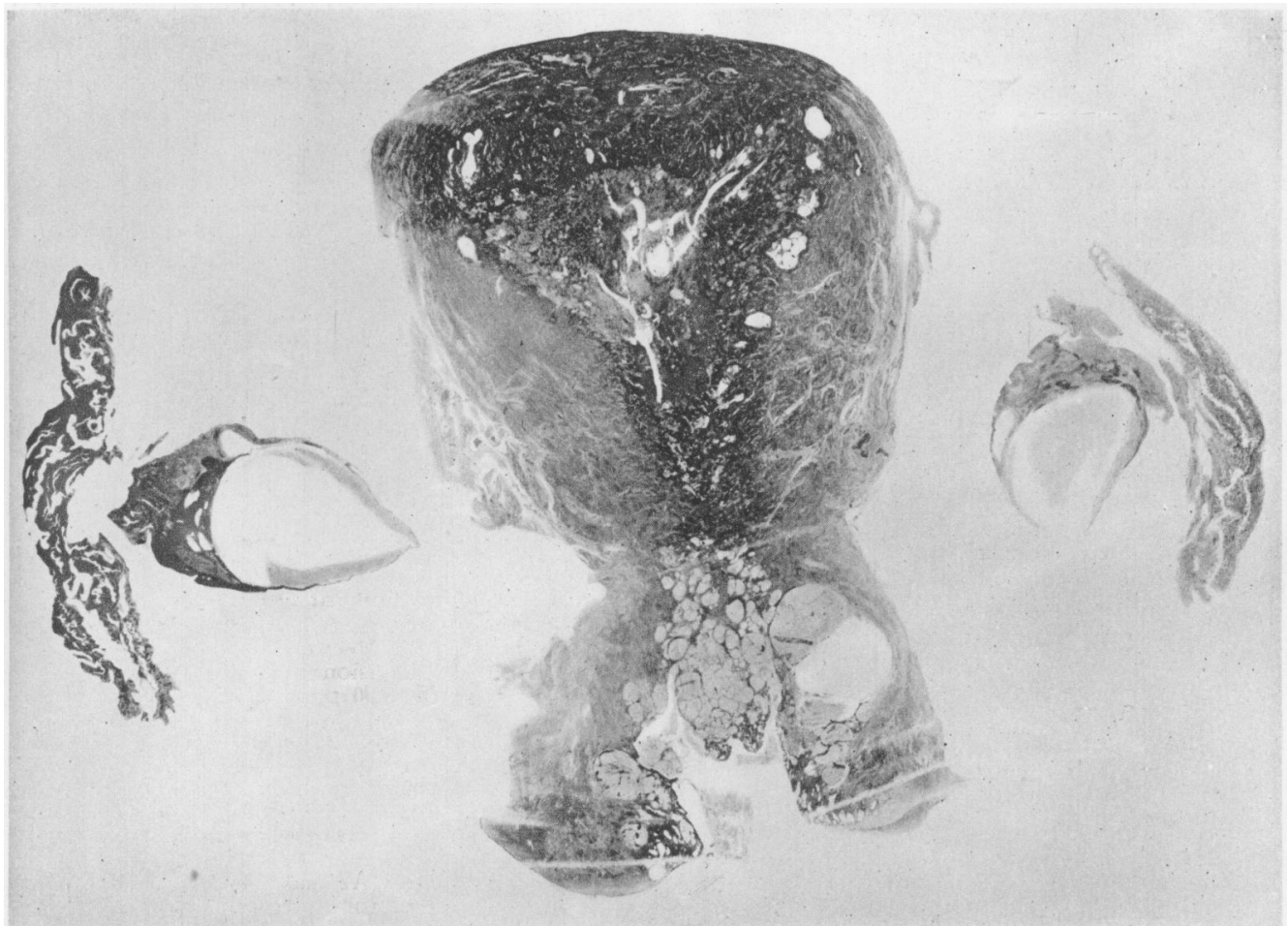

Fra. 5. - Uterus, tubes, and ovarles from woman, aged 53, 10 -para, with a long-standing history of menorrhagia. Admitted for fourteen weeks' continuod bleeding. The uterus has been cut out in the coronal plane complete in one glandular change in the endometrium; $(c)$ necrosis in the endonetrium (the darkly staining area in the lower part): ( $d$ ) Invasion of the muscle by endometrium (adenoinycosis); (e) proliferation and cystic change in cervical glands with polypoidal formation. There are bilateral cystic follicles in the ovaries. No corpora litea are present. Case of metropathia haemorrhagica. (Schroeder.)

A. DICKSON WRIGHT : VARICOSE ULCER

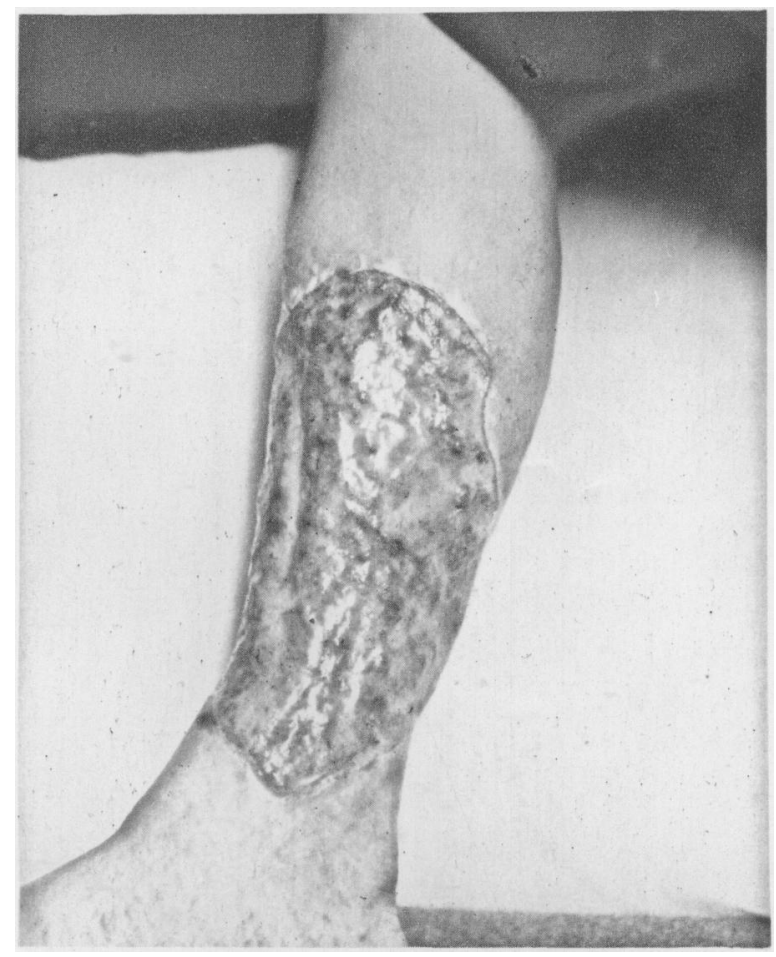

Fia. 1. - Mm. C. Iarge painful uloer of Ave years' duration.

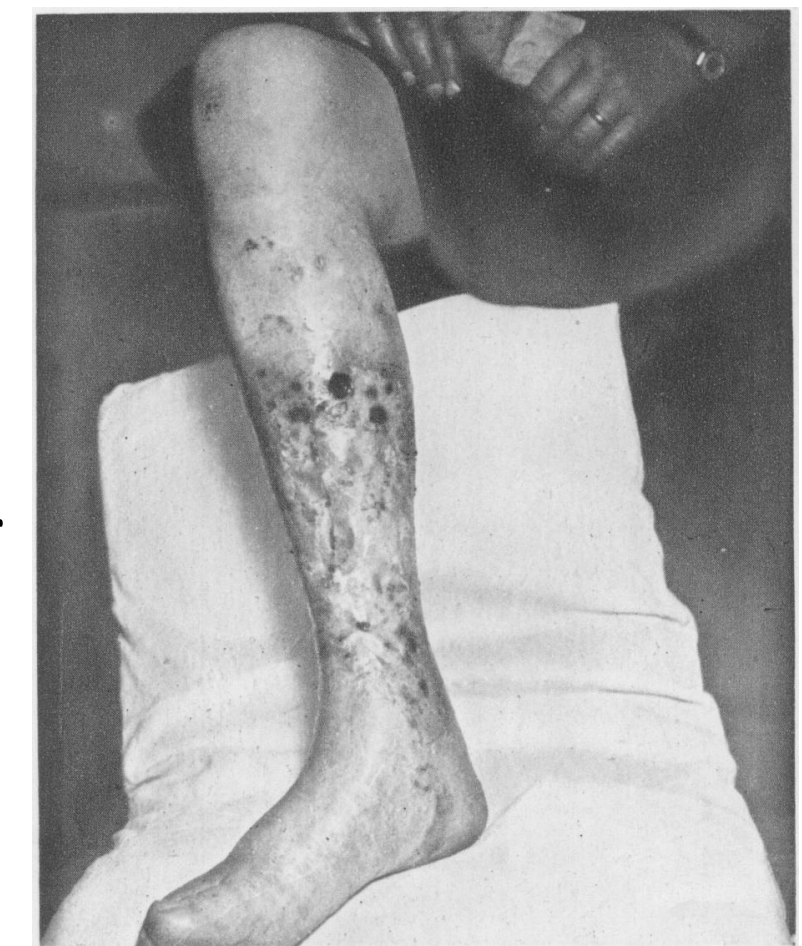

Fra. 2.-Mrs. C. Effoct of application of ten bandages in thirteen Fro. 2.- Mrs. C.
weoks' ikin graiting. 